\title{
Fibonacci-Hubbard Chain at Zero and Finite Temperatures
}

\author{
Sanjay Gupta ${ }^{\dagger}$, Shreekantha Sil ${ }^{\ddagger}$ and Bibhas Bhattacharyya ${ }^{\dagger \dagger}$ \\ ${ }^{\dagger}$ TCMP Division, Saha Institute of Nuclear Physics, 1/AF-Bidhannagar, \\ Calcutta - 700064, India. \\ $\ddagger$ Department of Physics, Vishwabharati, Shantiniketan, Birbhum \\ ${ }^{\dagger \dagger}$ Department of Physics, Scottish Church College, 1 \& 3, Urquhart Square, \\ Calcutta -700006
}

\begin{abstract}
We have studied finite-sized single band Hubbard chains with Fibonacci modulation for half filling within a mean field approximation. The ground state properties, together with the dc conductivity both at zero and non-zero temperatures, are calculated for such quasi-periodic Hubbard chains. While a reduction in the conductivity is found for strong electronic interaction or strong Fibonacci modulation, a competition between these two is observed to enhance the conductivity. The results at finite temperatures also illustrate some interesting features of such finite-sized systems.
\end{abstract}

PACS No.: 71.10.Fd, 71.23.Ft, 75.30.Fd 


\section{Introduction}

In recent years the study of the interplay of correlation and disorder in condensed matter systems has become a subject of great interest. Some recent experimental results, e.g. the metal-insulator transition (MIT) in two dimensional electron gas in Si-MOSFETs [1], suggest that there exists a competition between the electronic correlation and the disorder in such systems. Sheer complexity of the problem of considering simultaneous effects of both correlation and disorder attracted the attention of the researchers over the past few years. For example, the effect of Hubbard correlation in presence of random disorder has been studied by using quantum Monte Carlo techniques [2] and the bosonization [3]. Effect of electron-electron interaction on twodimensional finite clusters of disordered spinless fermions has been studied by diagonalization in a truncated Hartree-Fock (HF) basis [4]. Persistent current in disordered mesoscopic rings with spinless fermions, threaded by a magnetic flux, has been calculated by using exact diagonalization technique for finite-sized systems [5]. Similar systems have been studied by density matrix renormalization group (DMRG) [6] also. It seems interesting at this point to study the effect of electronic interaction in a quasi-periodic lattice. This not only interpolates between the extreme cases of full grown order and random disorder, but also gives some insight into the electronic properties of quasi-crystalline superlattices which are now realizable in the laboratory. Since the discovery of the quasicrystals [7], the electronic properties of these novel systems have been studied with great interest. The low temperature studies [8] of the electronic conductivity of the quasicrystalline 
systems pose some interesting questions. On the other hand, recent fabrication of quasicrystalline samples with local magnetic moments [9] raise the issue of dealing with the electronic correlation in quasicrystalline materials. Consequently, some works have already been carried out in this direction which include bosonization and weak-coupling renormalization group ( $\mathrm{RG}$ ) studies of interacting spinless fermions with diagonal Fibonacci modulation [10]. Hartree-Fock calculations for such Fibonacci-Hubbard chains have also been done [11] to identify the nature of the single particle spectrum and that of the wavefunctions in case of small electronic correlation. Recently, diagonal and off-diagonal Fibonacci modulations in an otherwise periodic Hubbard model has been studied by a weak-coupling RG, complemented by a DMRG calculation [12], which makes an attempt to identify gaps in the spin/charge sectors. However, to the best of our knowledge, a direct calculation of the electronic conductivity for such a system is yet to be done.

In view of this perspective we attempt a study of the competition between a quasiperiodic "disorder" (e.g. Fibonacci modulation in site potentials) and an on-site electronic correlation (e.g in the one band Hubbard model) in one dimension. The present study mainly concentrates on the calculation of the Drude weight [13] within a generalized Hartree-Fock approximation (GHFA) for studying the dc conductivity of such chains of finite lengths at half-filling. We have also studied the charge and spin density order parameters to characterize the ground state. It may be noted here that the study of the Drude weight may not be meaningful for a very large system with strong correlation or disorder [14]. For example, the strong on-site correlation could suppress the conductivity at half filling for a large chain. Therefore, our calculation is 
restricted to small finite systems in order to resolve the issue of competition between correlation and disorder. In fact previous Hartree-Fock studies of similar systems of large sizes [11] did not attempt the calculation of conductivity and were restricted to the limit of small correlation only (neglecting spin polarization) which is not expected to fully capture the essence of the competition between correlation and disorder.

In section II we define the Hamiltonian and explain the calculations in GHFA. Section III describes the results obtained from GHFA for zero temperature at half-filling. Section IV deals with the results at finite temperature. Section V summarizes the present work.

\section{The Model and the GHFA calculations}

\section{(i) Zero temperature calculation}

The Fibonacci sequence in any generation grows as:

$A B A A B A B A A B A A B A B A A B A B A A B A A B A B A A B A A B \ldots \ldots \ldots . . .$, growing with the generating rule where $A$ goes to $A B$ and $B$ goes to $A$ in the next generation. For an infinite Fibonacci chain the ratio of $A$ to $B$ is $(\sqrt{5}+1) / 2$, which is known as the golden mean.

We take a finite chain of $N$ sites, where $N$ is a Fibonacci number, in the form of a ring that is threaded by a flux (in units of basic flux quantum $\phi=$ $h c / 2 \pi e)[14]$; the Hamiltonian looks like:

$$
H=\sum_{i} \epsilon_{i} n_{i}+t\left(e^{i \phi} \sum_{i \sigma} c_{i \sigma}^{\dagger} c_{i+1 \sigma}+\text { h.c. }\right)+U \sum_{i} n_{i \uparrow} n_{i \downarrow},
$$

where $\epsilon_{i}$ is the site energy at the $i$-th site; it takes on the value $\epsilon_{A}$ or $\epsilon_{B}$ 
depending on whether it belongs to an $A$-type or a $B$-type site in the Fibonacci sequence. The number operator $n_{i \sigma}=c_{i \sigma}^{\dagger} c_{i \sigma}$ and $n_{i}=n_{i \uparrow}+n_{i \downarrow} ; t$ is the hopping integral between the nearest neighbour sites. The phase factor $\left(e^{i \phi}\right)$ appears because of the flux threaded by the ring. $U$ is the on-site Coulomb interaction.

On employing the GHFA the Hamiltonian is decoupled into up-spin and down-spin parts with the modified site energies for the up- and the down-spin electrons. The modified site energies are given by:

$$
\begin{aligned}
& \epsilon_{i \uparrow}^{\prime}=\epsilon_{i}+U<n_{i \downarrow}> \\
& \epsilon_{i \downarrow}^{\prime}=\epsilon_{i}+U<n_{i \uparrow}>
\end{aligned}
$$

This decoupled Hamiltonian is then diagonalized (in a self-consistent manner) for both the up and the down spin parts separately. We then calculate the ground state energy $E_{g}$ by summing the states up to the Fermi level for both up and down spins corresponding to a desired filling:

$$
E_{g}=\sum^{\mu} E_{n \uparrow}+\sum^{\mu} E_{n \downarrow}-U \sum_{i}<n_{i \uparrow}><n_{i \downarrow}>
$$

where $E_{n \sigma}$ 's are the single particle energy levels obtained by diagonalizing the decoupled Hamiltonian for the spin species $\sigma$.

The expressions for charge density wave (CDW) and spin density wave (SDW) correlation functions are given by

$$
\begin{aligned}
& C(q)=\frac{1}{N}\left|\sum_{p, m} e^{i q\left(R_{p}-R_{m}\right)}\left(n_{p}-1\right)\left(n_{m}-1\right)\right| \\
& S(q)=\frac{1}{N}\left|\sum_{p, m} e^{i q\left(R_{p}-R_{m}\right)}\left(n_{p \uparrow}-n_{p \downarrow}\right)\left(n_{m \uparrow}-n_{m \downarrow}\right)\right|
\end{aligned}
$$


respectively, where $q$ is the wave-vector of the corresponding density wave. $R_{p}$ and $R_{m}$ are position vectors of the $p$-th and the $m$-th sites respectively. The Drude weight which is a measure of the conductivity of the system is given by $[5,14,15]$ :

$$
D=-\frac{1}{N}\left(\frac{\partial^{2} E_{g}}{\partial \phi^{2}}\right)_{\phi=0}
$$

\section{(ii) Finite temperature calculation}

Upon diagonalization of the decoupled "independent electron" Hamiltonian one can calculate the partition function

$$
Z=\operatorname{Tr}\left[e^{-(\beta H-\mu N)}\right]
$$

where $\beta=1 / k T, k$ being Boltzmann's constant and $\mathrm{T}$, the temperature. This leads to direct calculations of several thermodynamic quantities. The Drude weight can be evaluated from

$$
D=-\frac{1}{N} \sum_{n}\left[\left(\frac{\partial^{2} E_{n \uparrow}}{\partial \phi^{2}}\right)_{\phi=0} \cdot \frac{1}{e^{\left(\frac{E_{n \uparrow}-\mu}{k T}\right)}+1}+\left(\frac{\partial^{2} E_{n \downarrow}}{\partial \phi^{2}}\right)_{\phi=0} \cdot \frac{1}{e^{\left(\frac{E_{n \downarrow}-\mu}{k T}\right)}+1}\right]
$$

\section{Results for zero temperature}

Our results are based on calculations for different sizes of the Fibonacci ring e.g. $N=34,55,89,233$ and 377 . We started our iteration with $\epsilon_{A}=0$ and $\epsilon_{B}=\epsilon>0$, with $U \geq 0$ the scale of energy being chosen by $t=1$. After achieving the self-consistent solution we find out $n_{i \uparrow}$ and $n_{i \downarrow}$ for each site $i$, which enables us to calculate the CDW and SDW order parameters defined 
in (3). $E_{g}$ is obtained as a function of $\phi$, by summing over states up to the Fermi level for both spins. The double derivative of $E(\phi)$ with respect to $\phi$ at $\phi=0$ gives the Drude weight. We have carried out the calculations for different system sizes with a fixed value of $\epsilon$ while varying $U$ and vice-versa.

It is interesting to observe how the competition between the correlation $(U)$ and quasiperiodic modulation $(\epsilon)$ decides the conductivity of the system (of a given size). It is also interesting to observe the dependence of this effect on system size. The competition between disorder and correlation is expected to be most prominent at half filling. This leads us to focus on this specific band-filling.

Fig. 1(a) shows the variation of the Drude weight with $(U / t)$ for different values of $\epsilon$ in a half filled chain of 34 sites. In this plot one can observe that the Drude weight is maximum at $U=0$ for $\epsilon=0$ and the scattering due to the Fibonacci "disorder" (for $\epsilon \neq 0$ ) tends to lower the conductivity compared to the case of $\epsilon=0$. However, in presence of the Fibonacci modulation in the site potentials (for $\epsilon \neq 0$ ), a competition between the correlation and the "disorder" enhances delocalization and, thereby, the conductivity. As a result of this, there should be a gradual rise in the Drude weight with increasing $U / t$. Such a competition between the Hubbard correlation and the aperiodic Fibonacci modulation is indeed showing up in the plots of the Drude weight in Fig. 1(a) with $\epsilon=1.0$ and 1.5 respectively. It can be argued on a heuristic ground that at $U \simeq 0$, there will be a considerable number of double occupancies (two electrons with opposite spins) that would favour to sit on A-type sites with lower site-energies for $\epsilon \neq 0$; this tendency of 
"pinning" would decrease the Drude weight from that of the case with $\epsilon=0$. An increase in the value of $U$ would tend to melt the "doublon"s and thereby undo the "pinning" effect. This would make the electrons more and more delocalized resulting in a rise of the Drude weight with increasing $U / t$ for $\epsilon \neq 0$. After attaining a maximum value at a certain $U / t$, which depends on the value of $\epsilon$ (apart from the system size), the Drude weight will again fall with increasing $U / t$ because of the usual suppression of the conductivity by the Hubbard correlation. Eventually the Drude weight becomes vanishingly small at a value of $U / t$, say $(U / t)_{c}$, which also depends on $\epsilon$ and system size. The general features noted above for the case of a ring of 34 sites are also observable in larger rings of 55, 89 sites (Fig.s 1(b) and 1(c)). Even for systems containing of as many as 233 or 377 sites (Fig.s 2(a) and 2(b)), we could easily observe similar features i.e. the qualitative dependence of Drude weight on $\epsilon$ and $(U / t)$ is independent of system size. We must make a comment here that the non-zero value of the Drude weight at half-filling is an effect of finite size. That is clearly indicated by the monotonic decrease in the peak value of the Drude weight with system size for a given value of $\epsilon$. It is interesting to note here that the value of $(U / t)$ for which the Drude weight is a maximum decreases with increasing disorder strength $(\epsilon)$. This feature, not clearly revealed for smaller system sizes can be prominently observed in Fig.s 2(a) and 2(b) where we find a shift of the peak in the Drude weight towards lower values of $(U / t)$ with increasing $\epsilon$. This is so prominent in Fig. 2(b) that for large enough $\epsilon$ we cannot recognize the gradual rise in the Drude weight. The effect of disorder takes over to suppress the delocalization due to the competition between $U$ and $\epsilon$. For smaller system sizes such effect 
will take place for larger $\epsilon$. On the other hand the value of $(U / t)_{c}$, where the Drude weight drops down to zero, depends on the system size. For a given value of $\epsilon$ it is observed that $(U / t)_{c}$ gradually decreases with the increase of the system size (Fig. 3). So the competitive effects of correlation and disorder show up in the conductivity of such systems of small sizes. The larger is the system size the weaker are the effects. Again for a ring of given length the competitive effect shows up for moderate values of $\epsilon$ and $U$. A very high value of correlation monotonically drives the system to an insulating phase; scattering due to strong disorder also suppresses (even if $U$ is reasonably large) the interplay of correlation and disorder (Fig. 2(b)). Such observations are in agreement with previous studies [12].

To investigate the nature of the ground state further we have plotted the spin and charge structure factors $S(q)$ and $C(q)$ (defined in (3)) respectively. In Fig.s 4(a) and 4(b) we have plotted $S(q)$ as a function of $q$ for different cases. In Fig. 4(a) we observe the sharp rise of the spin correlation function around $q=\pi$ for large $U$ (a value of $U$ when the Drude weight is vanishingly small) for system sizes $N=34,89$ and 233, at $\epsilon=0$. It is to be noted that precisely the point $q=\pi$ is missing for finite odd sized systems. In the "clean" limit antiferromagnetic SDW phase should settle, for larger $(U / t)$ which is revealed in the plot. We study the spin structure factor for $\epsilon \neq 0$ in Fig. 4(b) where we find smaller value of the $q=\pi$ peak and some wiggles at $q$ $\neq \pi$ due to an imperfect antiferromagnetic modulation caused by aperiodic site potentials. In presence of the Fibonacci modulation, there may arise a "pinning" tendency of the holes at special sites; hence the reduction in the 
value of $S(q)$ around $q=\pi$ and the wiggles at $q \neq \pi$. The effect is more pronounced at higher values of $\epsilon$. Plots of $S(q=\pi)$ as a function of $(U / t)$ (Fig.s 5(a) and 5(b)) shows that the antiferromagnetic SDW grows after crossing a certain $(U / t)$ that depends on the system size and on the value of $\epsilon$. These values $(U / t)$ match well with $(U / t)_{c}$ values obtained in the study of the Drude weight.

Fig.s 6(a), 6(b) and 6(c) show plots of the charge correlation function $C(q)$ against $q$ for different system sizes and different $U^{\prime} s$ at a reasonably large values of $\epsilon$. The quasiperiodic modulation generates peaks of different heights at incommensurate values of $q$. Density waves of such incommensurate (with lattice spacing) wave vectors get diminished in magnitude with increasing $U$. However the position of the peaks do not change. With the increase of the system size the prominent peaks do not disappear but get sharper and sharper. Moreover additional peaks of smaller magnitude appear to indicate excitation of new density waves for large system sizes. This is expected because of hierarchical aperiodic structures of the Fibonacci generations.

In explaining the decrease in the Drude weight with increasing "disorder" we conjectured the formation of "pinned" holes and "doublons". These "pinning" would follow the Fibonacci modulation and would be partly modified by the probability of hopping between adjacent sites of comparable site energies. That this really takes place is evident from the plot of the density profile against the site index (Fig. 7) for the 34-site ring. On the other hand $A$ type sites favour retaining "doublons" (with average occupancy greater than 1). The $A A$ pairs, on the contrary, show a lesser preference for the 
"doublons" to facilitate nearest neighbour hopping which lowers the energy.

\section{Finite temperature calculation of Drude weight}

The Drude weight, as calculated from (5), is plotted against the temperature in Fig.s 8(a), 8(b), 9(a) and 9(b) for system sizes $N=34,55,89$ and 233 respectively. Cases with zero and non-zero values of $\epsilon$ and $U$ are compared in the graphs. Since the effect of correlation is expected to play an important role at the half-filling, the 34-site system is studied at this special filling $\left(=\frac{17}{34}\right)$ while for the 55,89 and 233 -site chains we concentrate on the fillings $n=28 / 55,44 / 89$ and $116 / 233$ respectively which are the closest possible cases to (i.e. one particle away from) half-filling.

Fig. 8(a) shows the variation of the Drude weight against the temperature $(k T)$ for system size $N=34$. In absence of correlation $(U=0)$ the Drude weight starts from its maximum value (i.e. $4 t / \pi$ ) at half-filling for the periodic case $(\epsilon=0)$. There is a gradual fall in the Drude weight with the rise in the temperature. This is because the thermal excitations tend to populate the higher levels which diminish the current generated by the breaking of the time-reversal symmetry due to the external field. The rate of decrease of $D$, however, is too small to be clearly detected in the graph. However, in the presence of the "Fibonacci disorder" $(\epsilon \neq 0)$ the scattering effect takes over and this makes the conductivity fall in a much sharper fashion with the increase of the temperature. On the other hand, at $T=0$,

the Hubbard correlation introduces a Mott gap $[16,17]$ at the middle of the 
band below which all levels (i.e. the lower band) are occupied at half-filling. Therefore, the conductivity drops down to a much lower value (non-zero for a small finite system) in the case of $U \neq 0$ as compared to the non-interacting system. As the temperature increases, the excitations of the electrons take place across the Mott gap to populate the upper band. This results in an enhanced conductivity of the system. In the limit of very large temperature the Drude weight asymptotically approaches to that of the non-interacting case $(\epsilon=U=0)$ from below. Simultaneous presence of aperiodic "disorder" and the Hubbard interaction leads to a competition that reduces the Mott gap. This is why the value of the Drude weight for $\epsilon \neq 0, U \neq 0$ is slightly higher than that in the case of $\epsilon=0, U \neq 0$. Now the Drude weight falls with the rise in temperature, the rate of fall being primarily dominated by the effect of disorder.

More or less similar pictures evolve for other system sizes (Fig.s 8(b),9(a) and 9(b)). However, a few notable differences are there. Firstly, the effect of the correlation becomes more pronounced with increasing system-size. This is why the Drude weight is vanishingly small for $U / t=1.7\left(>(U / t)_{c}\right)$ for system sizes as large as $N=89$ and 233 irrespective of the presence of disorder (i.e. for both the cases of $\epsilon=0$ and $\epsilon \neq 0$ ). Secondly, the rate of fall of the Drude weight (with temperature) is enhanced for larger systems for which the effect of disorder-induced scattering is stronger. It is noteworthy that in Fig. 8(b) for $N=55$ the Drude weight initially falls and then increases with the rise in the temperature for the ordered case with large correlation $(\epsilon=0, U / t=1.7)$. This feature is in contradiction with the case depicted in Fig. 8(a) for $N=34$. This is because we have considered the 
precisely half-filled case for $N=34$ while the case of $N=55$ corresponds to a case of one particle away from the half-filling (filling $=28 / 55$ ). So, even in presence of the Mott gap (comparable to the finite-size gaps) the system behaves like a metal at low temperatures. Therefore, for the initial rise in temperature we observe a slow decrease in the Drude weight in Fig. 8(b). For higher temperatures the effect of thermal excitations of electrons across the Mott gap dominates. This results in the ultimate rise in the Drude weight in this region. It is to be noted that this feature will gradually vanish with increasing system size for which the finite-size gaps become smaller and smaller in comparison with the Mott gap. Indeed, this feature is merely detectable in Fig. 9(a) for $N=89$ while it completely disappears for $N=233$ (Fig. 9(b)). The fact that the effect of the Mott gap becomes more dominant for larger systems (for the same value of the Hubbard parameter $U$ ) is also evident from the case of $\epsilon=0.5, U=1.7$ in Fig. 9(b) i.e. for $N=233$. Here we find a small rise in the Drude weight with temperature even in presence of disorder, a feature not observable in Fig.s 8(a), 8(b). For larger systems the introduction of a small disorder essentially drives the system to become an insulator which is can be sharply contrasted with that of a Mott insulator. In the regime of higher temperatures we find that the conductivity of the disorder-driven insulator $(\epsilon=0.5 U / t=1.7)$ is less than that of the correlation-driven (Mott) insulator $(\epsilon=0, U / t=1.7)$ by several orders of magnitude. This is, indeed, a very good illustration of differentiating the two types of insulators- namely, disorder-driven insulator and Mott insulator.

\section{Conclusion}


Summarizing, we have studied finite-sized Fibonacci-Hubbard chains at half filling (or one particle away from half-filling). In the present work, within the mean field approach of GHFA, we calculate the ground state properties e.g. the spin and charge correlation functions, and the dc conductivity both at zero and finite temperatures. We find that the dc conductivity, as obtained from the Drude weight $[5,14]$, shows a strong dependence on the competition between the Fibonacci modulation and the Hubbard correlation. This tends to enhance the conductivity at zero temperature in the regime of intermediate coupling. However, in the asymptotic cases of very weak and strong Hubbard correlations (in presence of a reasonable modulation in the site potentials), the conductivity is reduced due to diagonal aperiodicity (driving towards a Fibonacci modulated charge ordering) and electronic correlation (bringing in antiferromagnetic fluctuations) respectively [12]. The value of $(U / t)_{c}$, where the conductivity of the system drops down to zero, decreases with the system size. This suggests that the true effect of competition of the correlation and disorder could be appreciably observed in systems of smaller size (typically 100 sites). The SDW correlation strongly grows with Hubbard correlation at $q=\pi$ at half-filling. This indicates the dominance of antiferromagnetic instabilities at large $U$ values. The charge correlation $C(q)$ shows spikes characteristic of Fibonacci modulation. This charge modulation although reduced by the presence of finite $U$ does not go off even at a value of $U$ for which anti-ferro SDW starts dominating. There appear more and more characteristic spikes in $C(q)$ as the system size increases. However, the spikes observed for smaller system sizes never disappear. This suggests that the system captures the self similar pattern of the Fibonacci hierarchy 
and this is not masked by correlation. At finite temperatures, the Drude weight slowly decreases with rise in the temperature, in the periodic limit in absence of correlation. The presence of Hubbard correlation opens up a Mott gap which quenches the conductivity at low temperatures. Therefore, with the increase of temperature, there are excitations of electrons across the Mott gap to give rise to an initial increase in the conductivity. This semiconductor-like behaviour is in sharp contrast to the periodic case. On the other hand, the scattering induced by the Fibonacci disorder makes the picture completely different. In presence of Fibonacci modulation alone the Drude weight sharply decreases with temperature. This feature shows up even in presence of the Hubbard interaction for smaller system sizes. However, for larger systems at very low temperatures (in presence of correlation) the Mott gap reduces the conductivity to such a small value that the disorderdriven fall in the Drude weight is almost invisible. In the high temperature region the behaviour of the Mott insulator and that of the disorder-driven insulator are in sharp contrast to each other. The results obtained for a 55site system (studied for one particle away from half filling) indicate that the effect of variation in the band filling could give rise to some notable features. As revealed in the present study, the competition between electronic correlation and diagonal aperiodicity leads to interesting effects in the properties of such systems at both $T=0$ and $T \neq 0$ for smaller system sizes. It is, therefore, worthwhile to make further studies of such systems. For example, it would be interesting to see the effect of different types of quasiperiodic modulations and the effect of external fields in such systems, using similar mean-field approaches as well as techniques beyond the mean-field approxi- 
mations $[2,12]$. Also a detailed study of the effect of band filling away from the half filled case would be an interesting one at finite temperatures. 


\section{References}

[1] E. Abrahams, S. V. Kravchenko and M. P. Sarachik, Rev. Mod. Phys. 73, 251 (2001), and references therein.

[2] A. W. Sandvik, D. J. Scalapino and P. Henelius, Phys. Rev. B 50, 10474 (1994).

[3] T. Giamarchi, P. Le Doussal and E. Orignac, Phys. Rev. B 64, 245119 (2001).

[4] T. Vojta, F. Epperlein and M. Schreiber, Phys. Rev. Lett. 81, 4212 (1998).

[5] G. Bouzerar, D. Poilblanc and G. Montambaux, Phys. Rev. B 49, 8258 (1994).

[6] P. Schmitteckert et. al., Phys. Rev. Lett. 80, 560 (1998); P. Schmitteckert et. al., Phys. Rev. Lett. 81, 2308 (1998).

[7] D. Shechtman et. al., Phys. Rev. Lett. 53, 1951 (1984).

[8] D. Mayou et. al., Phys. Rev. Lett. 70, 3915 (1993); T. Klein et. al., Phys. Rev. Lett. 66, 2907 (1991); B. D. Biggs, S. J. Poon and N.Munirathan, Phys. Rev. Lett. 65, 2700 (1990).

[9] T. J. Sato et. al., Phys. Rev. Lett. 81, 2364 (1998); T. J. Sato et. al., Phys. Rev. B 61, 476 (2000). 
[10] J. Vidal, D. Mouhanna and T. Giamarchi, Phys. Rev. Lett. 83, 3908 (1999). J. Vidal, D. Mouhanna and T. Giamarchi, Phys. Rev. B 65, 014201 (2001).

[11] H. Hiramoto, J. Phys. Soc. Jpn. 59, 811 (1990). N. Fujita and K. Niizeki, Mat. Sci. Eng. A 294, 560 (2000)

[12] K. Hida, Phys. Rev. Lett. 86, 1331 (2001).

[13] W. Kohn, Phys. Rev. 133, A171 (1964).

[14] S. Gupta, S. Sil and B. Bhattacharyya, Phys. Rev. B 63, 125113 (2001); ibid. 64, 1

[15] R. M. Fye et. al., Phys. Rev. B 44, 6909 (1991); D. J. Scalapino, S. R. White and Z. Shoucheng, ibid. 47, 7995 (1993).

[16] J. Hubbard, Proc. Roy. Soc. A 276, 238 (1963).

[17] N. F. Mott, Metal-Insulator Transitions, chapter 4, Taylor \& Francis Ltd. (London) (1974). 


\section{Figure Captions}

Fig. 1. Plot of the Drude weight $D$ (at zero temperature) vs. $U / t$ for different values of $\epsilon$ for a Fibonacci-Hubbard ring of (a) 34 sites (with 34 particles), (b) 55 sites (with 56 particles) and (c) 89 sites (with 88 particles). A finite value of $D$ for low $U / t$ at half-filling is a finite-size effect. For non-zero values of $\epsilon$ the competition between Hubbard correlation and the diagonal Fibonacci modulation is clearly seen from low to intermediate values of $U / t-$ upto a value $(U / t)_{c}$.

Fig. 2. Plot of the Drude weight $D$ (at zero temperature) vs. $U / t$ for the Fibonacci-Hubbard chain of (a) 233 sites (with 232 particles) and (b) 377 sites (with 376 particles) for different values of $\epsilon$. The shift of the peak in the Drude weight towards $U / t=0$ is clearly visible for higher values of $\epsilon$.

Fig. 3. Plot of $(U / t)_{c}$ vs. $N$, the system size (at/ one particle away from half-filling).

Fig. 4. Plot of the SDW correlation function $S(q)$ (at zero temperature) as a function of the wave-vector $q$ for different systems sizes (a) in absence of disorder $(\epsilon=0)$ and (b) in presence of Fibonacci modulation $(\epsilon=1.5)$. Value of the Hubbard correlation $U=3.0$ (the scale of energy is given by $t=1.0)$. The peak at $q=\pi$ shows antiferromagnetic spin modulation induced by large $U$. This correlation is reduced by the presence of Fibonacci "disorder" and also there appear wiggles at $q \neq \pi$. Such wiggles are merely 
visible in (b).

Fig. 5. Plot of $S(q=\pi)$ (at zero temperature) vs. $U / t$ for different values of $\epsilon$ for the Fibonacci-Hubbard chain of (a) 34 sites, and (b) 89 sites. For the Hubbard model $(\epsilon=0) S(q=\pi)$ grows with $U / t$ at half-filling. The sharp rise in $S(q=\pi)$ follows after a certain value of $(U / t)$ which is in close agreement with the $(U / t)_{c}$ observed in Fig. 1.

Fig. 6. Plot of the CDW correlation function $C(q)$ (at zero temperature) vs. the wave-vector $q$ for the Fibonacci-Hubbard chain of (a) 34 sites, (b) 89 sites, and (c) 233 sites. There are characteristic peaks for a large value of the "disorder" $(\epsilon=1.5)$ which do not disappear with the increase in the correlation parameter $U$.

Fig.7. Plot of the particle density $n_{i}$ against the site index $i$ for the FibonacciHubbard chain of 34 sites at zero temperature at half-filling with $\epsilon=1.0$ and $U=3.0$ (in unit of $t$ ). The density profile shows formation of a charge density modulation which closely resembles the Fibonacci pattern.

Fig. 8. Plot of the Drude weight $D$ vs. the temperature $T$ (scaled up by the Boltzmann constant $k$ ) for the Fibonacci-Hubbard chain of (a) 34 sites (at half-filling), and (b) 55 sites (one particle away from half-filling) for different choices of $\epsilon$ and $U$ (scale of energy $t=1.0$ ).

Fig. 9. Plot of the Drude weight $D$ vs. the temperature $T$ (scaled up by the 
Boltzmann constant $k$ ) for the Fibonacci-Hubbard chain of (a) 89 sites, and (b) 233 sites (for one particle away from half-filling for both the cases) for different choices of $\epsilon$ and $U$ (scale of energy $t=1.0$ ). 


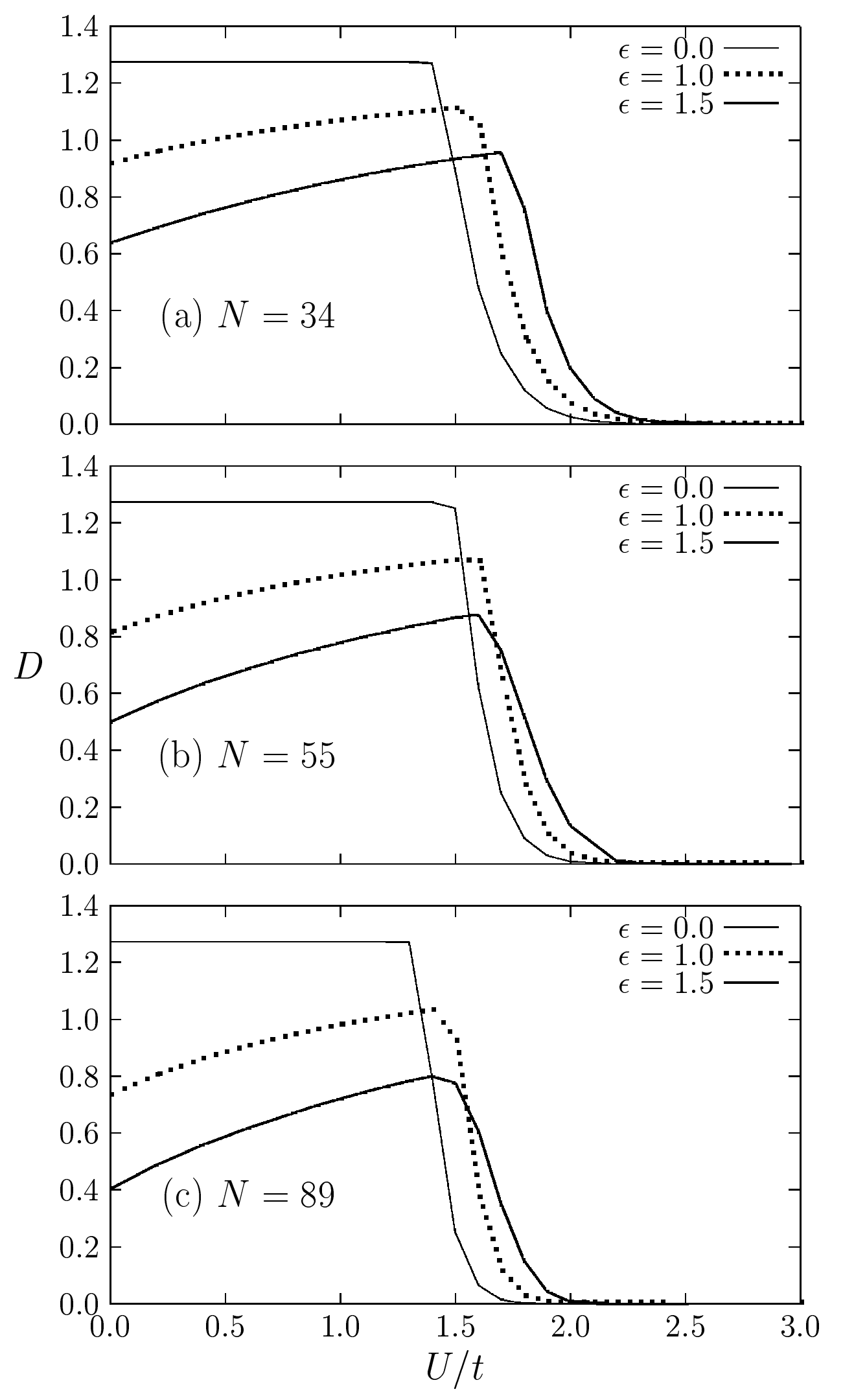




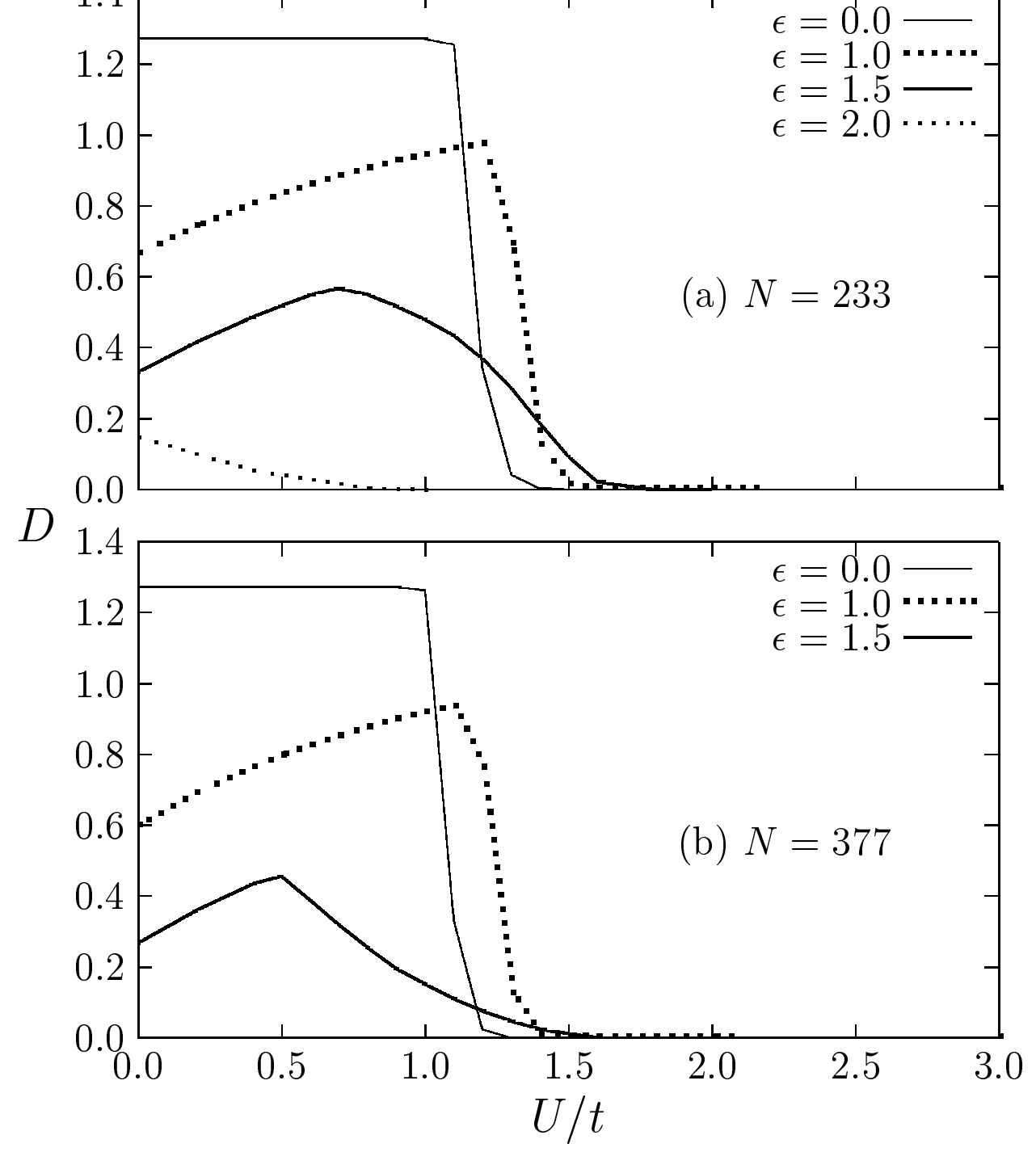




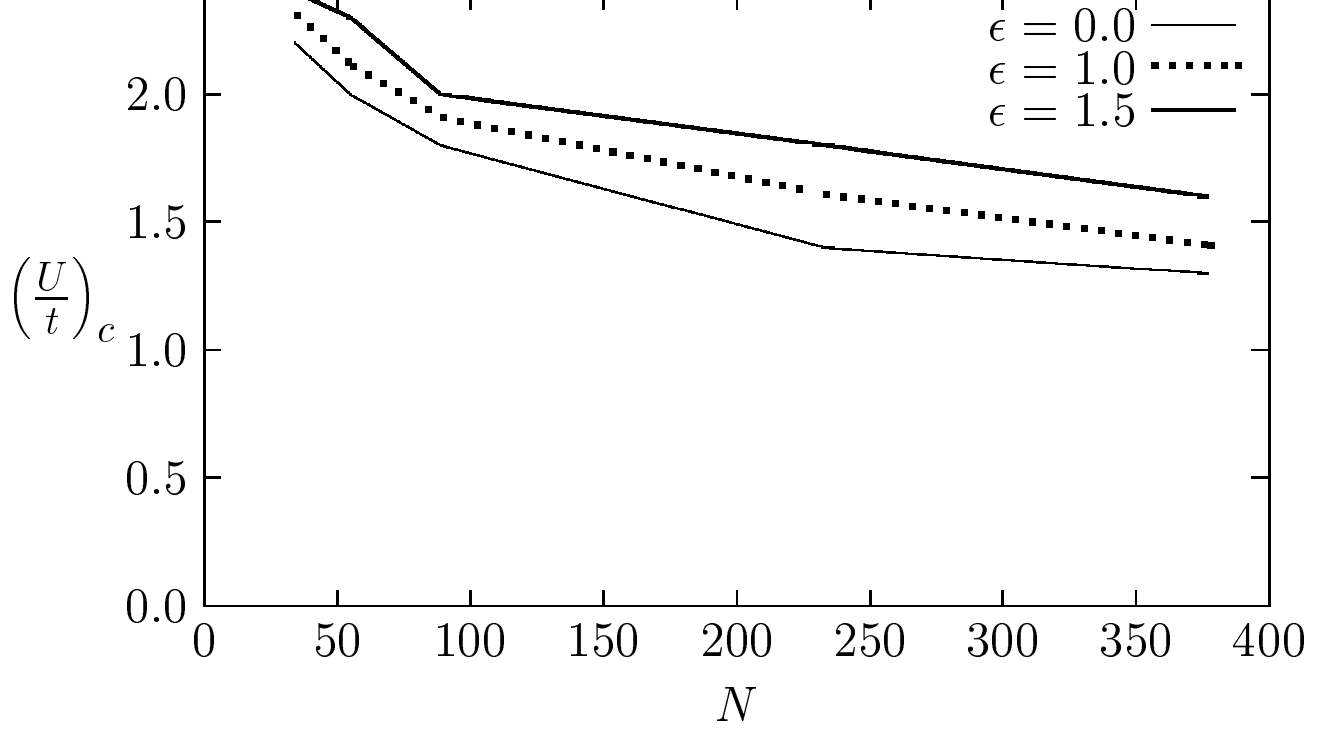




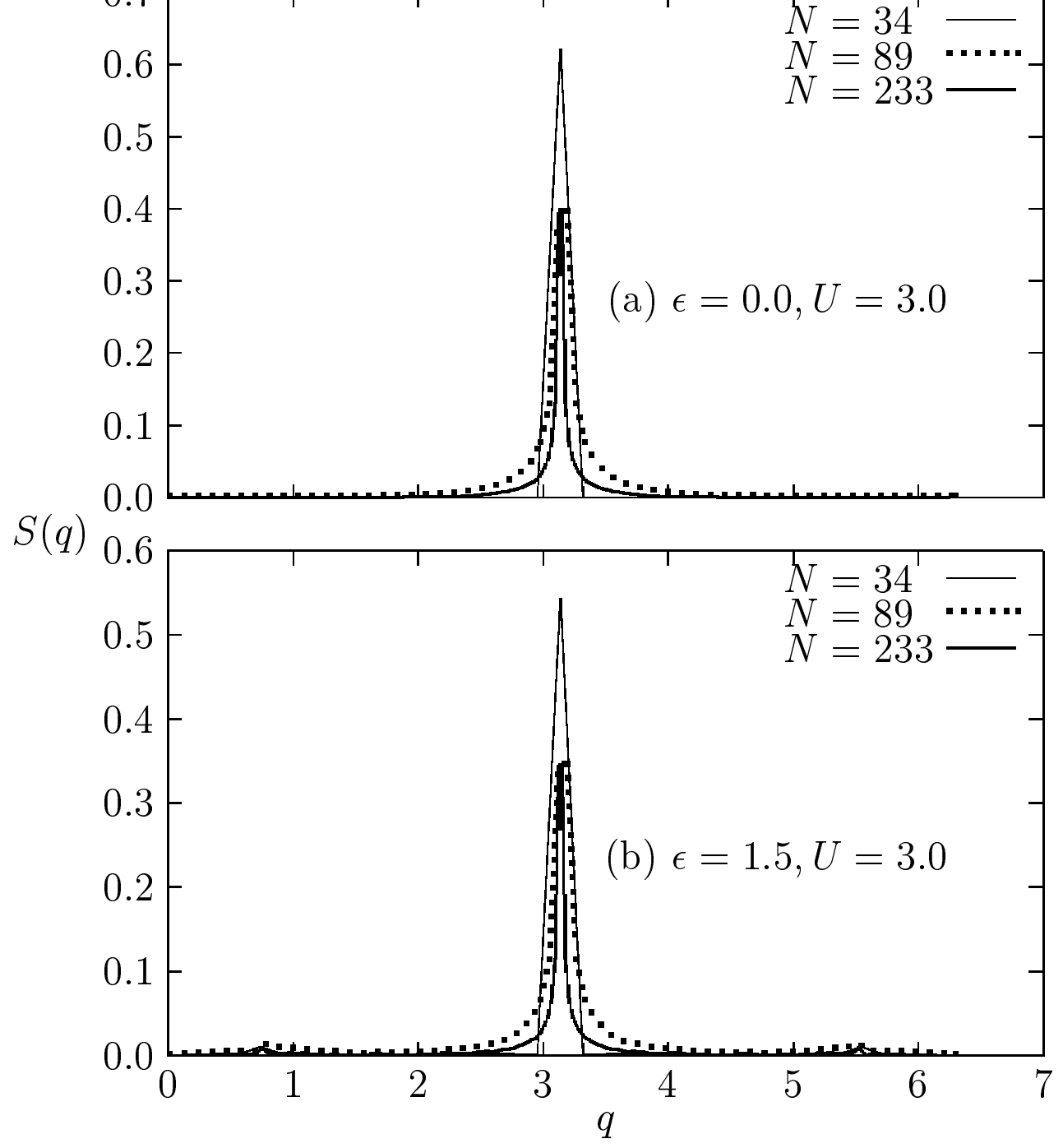




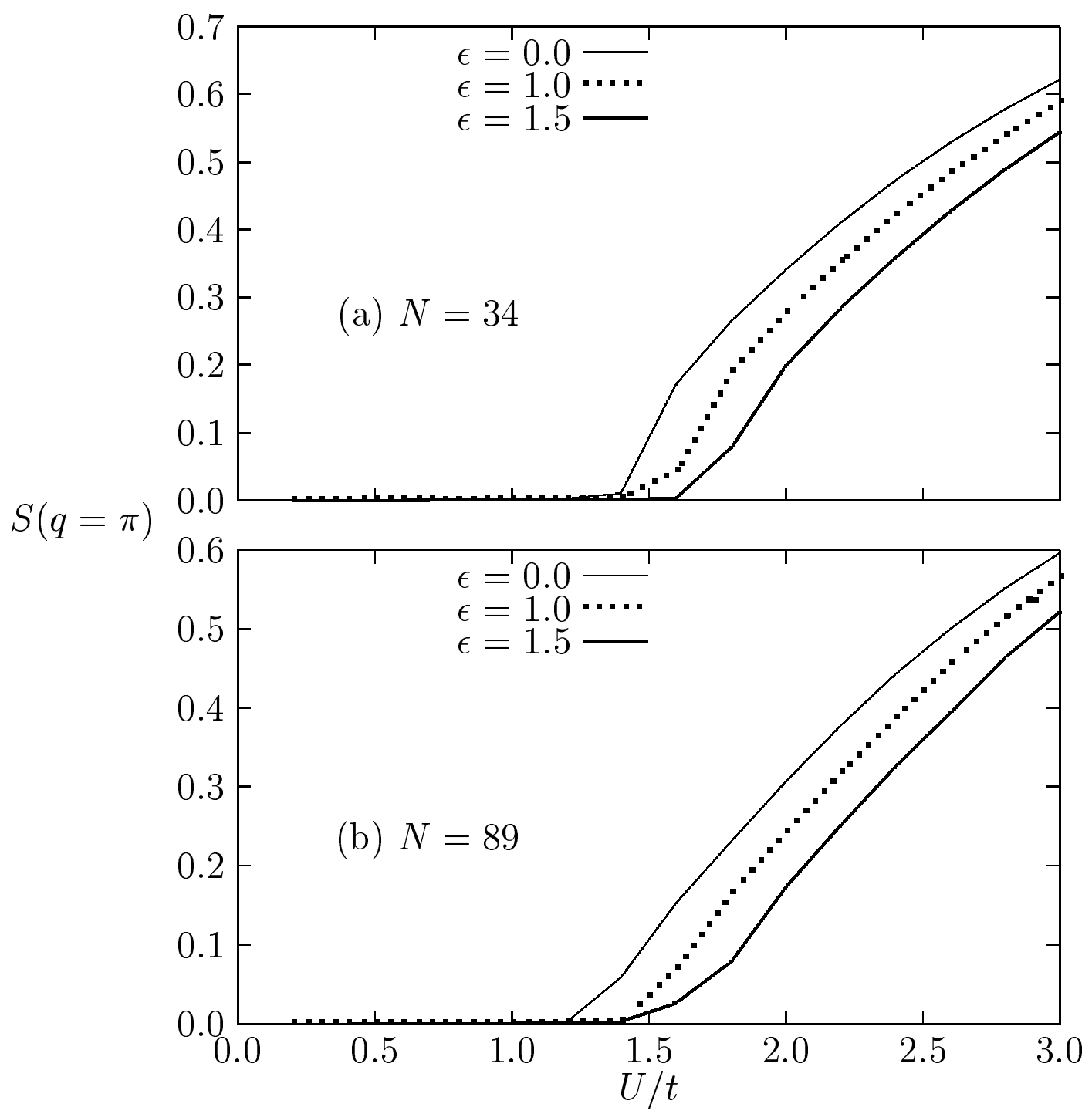




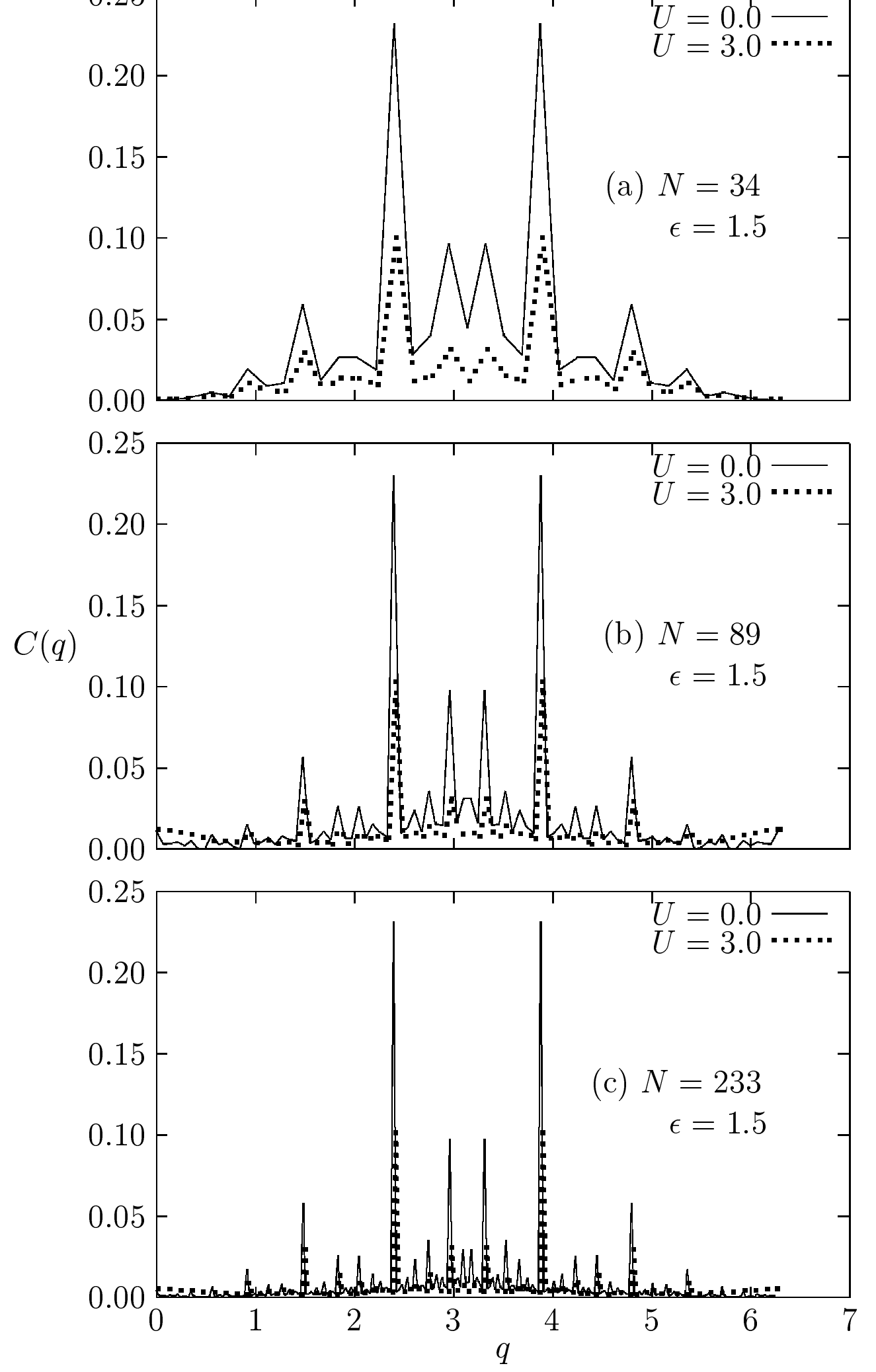




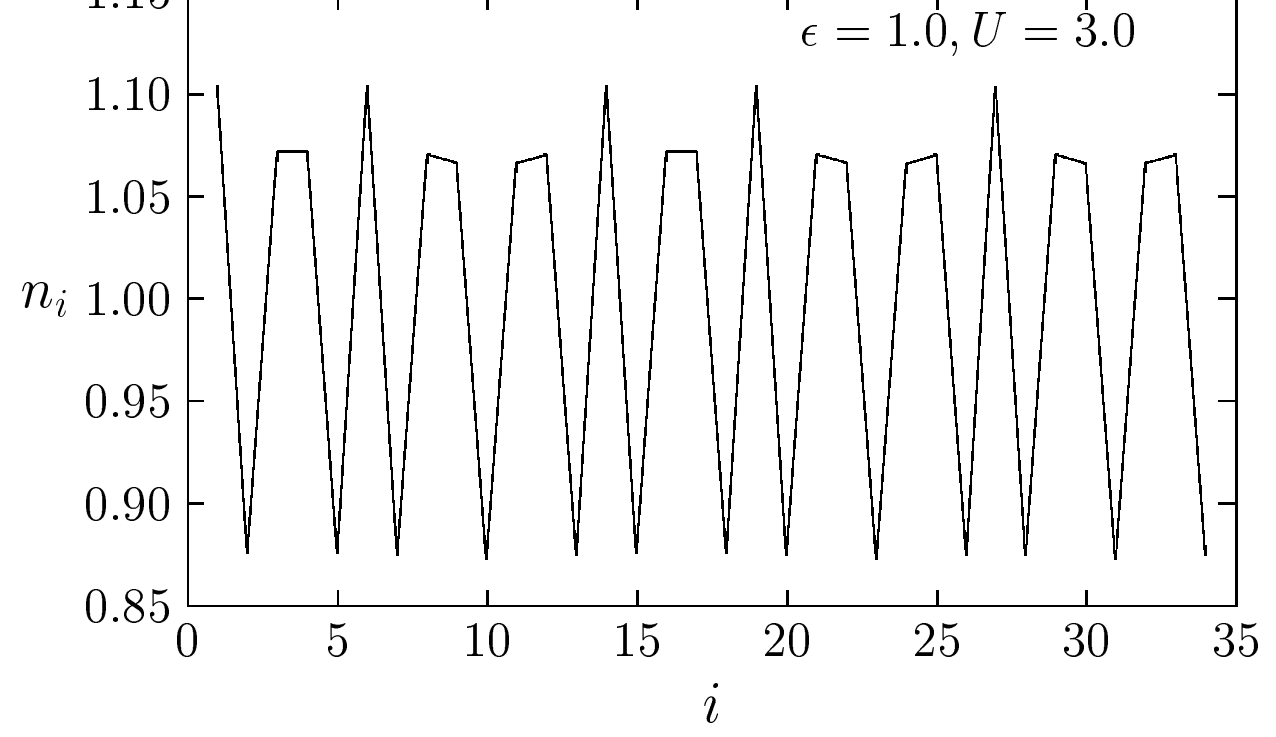



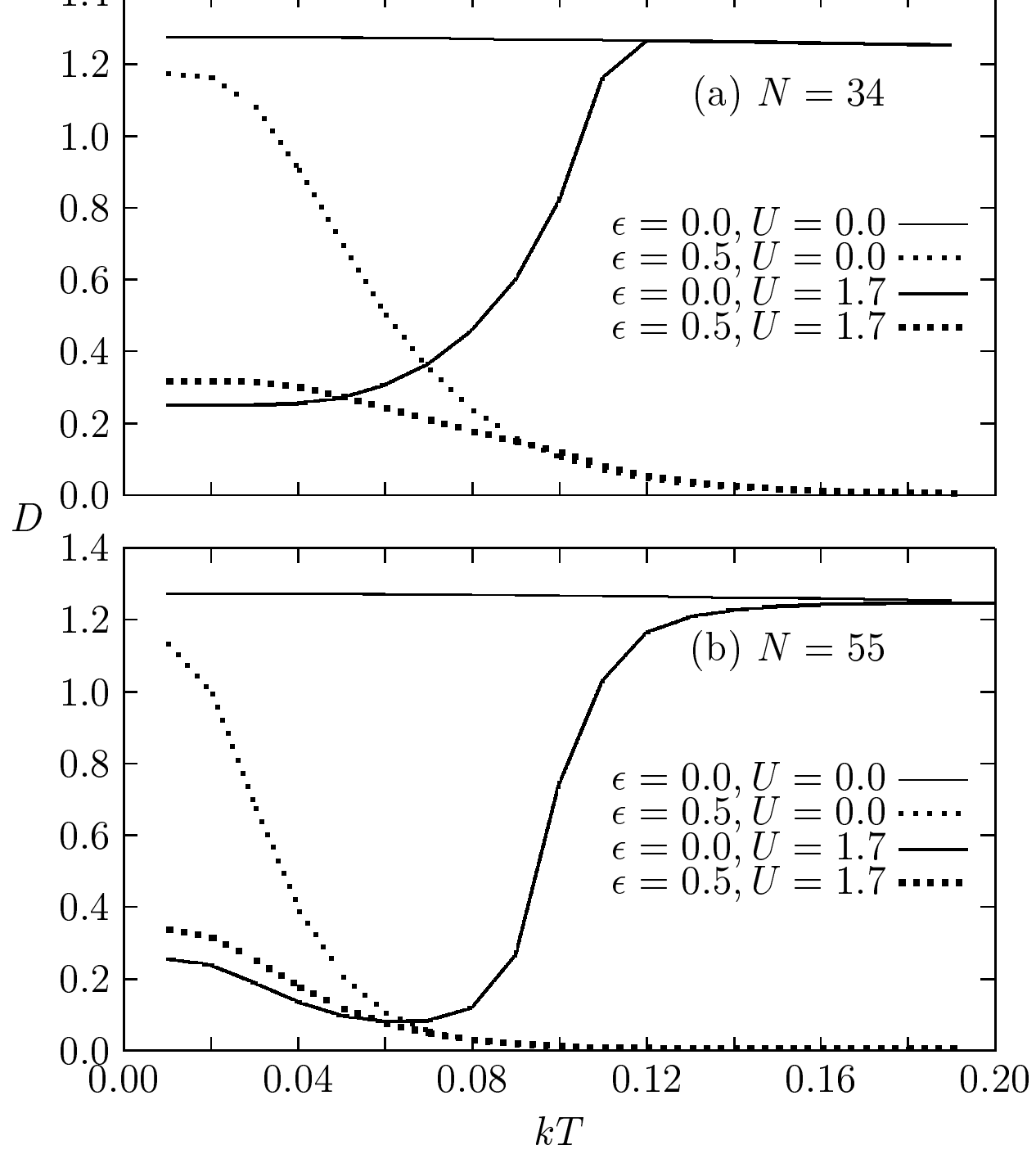


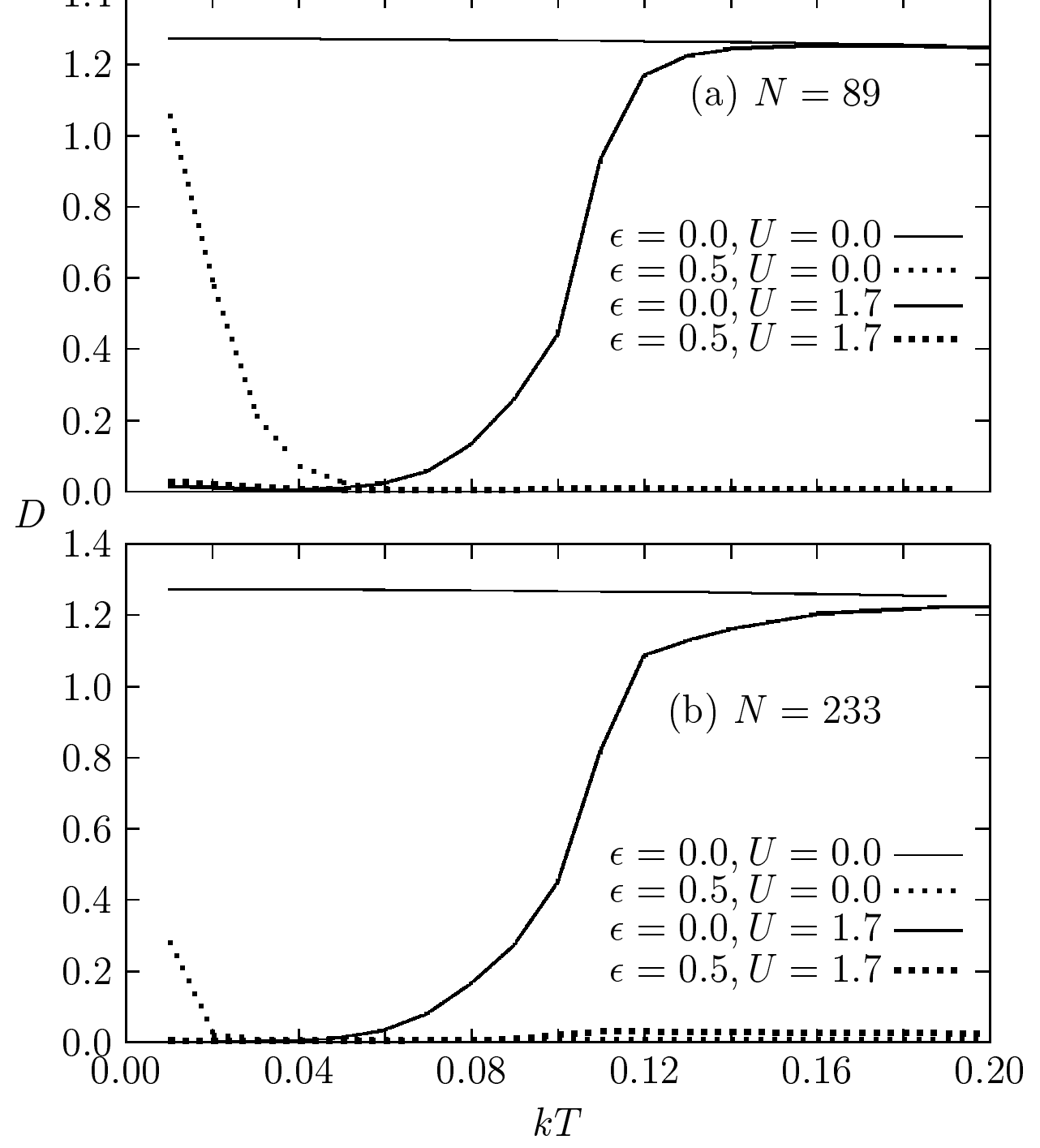

